\title{
Development and analysis of a longitudinal and torsional type ultrasonic motor with two stators
}

\author{
Youping Yi ${ }^{a, *}$, Wolfgang Seemann ${ }^{b, 1}$, Rainer Gausmann ${ }^{c, 2}$, Jue Zhong ${ }^{\text {a,3 }}$ \\ ${ }^{a}$ College of Electrical and Mechanical Engineering, Central South University, 410083 Changsha, China \\ ${ }^{\mathrm{b}}$ Institut für Technische Mechanik, Universität Karlsruhe (TH), 76128 Karlsruhe, Germany \\ c AG Maschinendynamik, Universität Kaiserslautern, 67653 Kaiserslautern, Germany
}

Received 20 August 2004; received in revised form 17 February 2005; accepted 17 March 2005

Available online 18 April 2005

\begin{abstract}
In this paper, a new longitudinal and torsional type ultrasonic motor with two stators is presented and investigated. Normally, such a motor consists of one rotor and one stator, and two types of transducers that are longitudinal PZT and torsional PZT are used to generate the desired elliptical locus on the stator surface. The operating frequency is at the resonance frequency of torsional transducer. In order to enhance the efficiency of the motor, however, the resonance frequencies of both transducers should be closed to each other. For the purpose of matching the resonance frequencies, a symmetrical structure is adopted in design of the motor. Furthermore, two rings are added to the stators in order to adjust the resonance frequencies of these two transducers. A finite element model is developed and ANSYS software is used to analyze the resonance frequencies of longitudinal vibration and torsional vibration as well as optimize the motor geometry. According to the FE results, an experimental prototype is fabricated and the experimental results agree well with the theoretical predictions.
\end{abstract}

(C) 2005 Elsevier B.V. All rights reserved.

Keywords: Ultrasonic motor; Vibration; Transducer; PZT; Resonance frequency

\section{Introduction}

Ultrasonic motors have excellent performance and many useful features such as high holding torque, high torque at low speed, quiet operation, simple structure, compactness in size and no electromagnetic interferences. It may be widely used as actuators for robots,

\footnotetext{
${ }^{*}$ Corresponding author. Tel.: +86 731887 6163; fax: +86 731871 0201.

E-mail addresses: yyp@mail.csu.edu.cn (Y. Yi), seemann@itm.unikarlsruhe.de (W. Seemann), rainer.gausmann@gmx.net (R. Gausmann), jzhong@mail.csu.edu.cn (J. Zhong).

${ }^{1}$ Tel.: +49 721608 6824; fax: +49 7216086070 .

2 Tel.: +49 203486 2611; fax: +49 1212512112147 .

${ }^{3}$ Tel.: +86731887 9352; fax: +867318710201.
}

cameras, precise positioning devices, machines in space and medical treatment devices [1,2].

A longitudinal and torsional type ultrasonic motor uses longitudinal and torsional PZT transducers to generate the desired elliptical orbit of the stator surface. Compared to a traveling wave ultrasonic motor, this motor belongs to a standing wave motor and can produce larger output torque. Since Ueha [3] firstly proposed such motor in 1980s, more attentions have been attracted in this motor and various attempts to improving its performance have been reported in Refs. [4-11]. Generally, a longitudinal and torsional type ultrasonic motor operates at the resonance point of torsional vibration to achieve a long stroke in tangential direction and obtain a high efficiency. However, the normal force generated by longitudinal vibration is also important to enhance the output torque. It generates frictional force 
to drive the stator at the interface between rotor and stator. Normally, there are two ways to increase the normal force: one is to enlarge the contact area of rotor and stator; the other is to make the longitudinal and torsional transducer have an identical resonance frequency. Due to the different sound propagation speed of longitudinal and torsional vibrations within a motor, however, it is difficult to match the resonance frequency of longitudinal and torsional vibrations [12]. In order to improve such motor, several methods to matching the resonance frequencies of longitudinal and torsional vibrations have been proposed in Refs. [13-16].

In this paper, a new longitudinal and torsional type ultrasonic motor using one rotor and two stators is proposed and investigated. For the purpose of enlarging the contact area, the rotor is placed in the middle of the motor and is driven by two stators. A method, adding two rings to the outside circumference of two stators respectively, is proposed to adjust the resonance frequencies of longitudinal and torsional vibrations. A FE model of stator is developed to analyze the resonance frequencies of longitudinal vibration and torsional vibration as well as optimize the motor geometry. Finally, a prototype of the present motor is fabricated according to the FE results.

\section{Working principle}

The structure of present motor is shown in Fig. 1. The present motor consists of one rotor and two stators, in which the inverse piezoelectric effect is used to excite two orthogonal vibrations modes: one is longitudinal vibration excited by longitudinal transducer L-PZT and the other is torsional vibration excited by torsional transducer L-PZT. For the operation of the motor, the first mode of longitudinal vibration and the first mode of torsional vibration are used (see Fig. 1). When these two vibrations are excited, two stators extend and contract simultaneously. By pressing two stators against

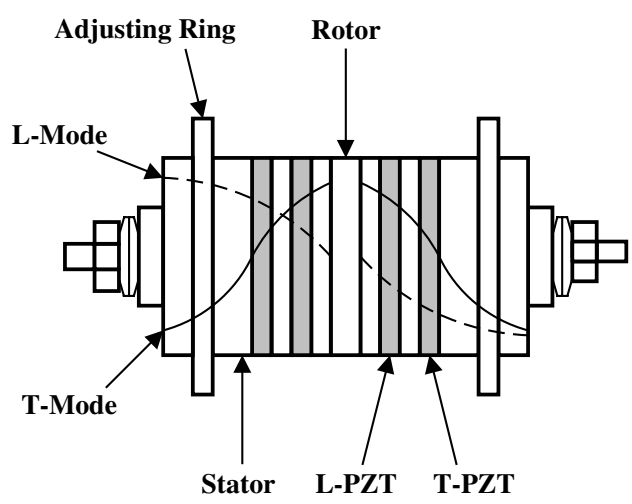

Fig. 1. Sketch of a longitudinal and torsional ultrasonic motor with two stators. the rotor with a certain pre-load, friction forces between rotor and stator occur and lead to the rotor rotation. Obviously, the contact area between rotor and stator of the present motor is two times as large as the previous hybrid ultrasonic motor with one stator. Therefore, the present motor can produce much larger friction force on the contact areas. In addition, two adjusting rings are added to the outside circumference of two stators to support motor and to adjust the resonance frequencies of the vibrations excited in stator.

By applying the $\mathrm{AC}$ voltages $V_{\mathrm{L}}$ and $V_{\mathrm{T}}$, the first longitudinal mode and the first torsional mode can be excited in the stator, respectively. Considering that the excitation is harmonic in time, the displacement of a point on stator surface is given by

$U_{\theta}(t)=A_{1} \sin \omega t$

$U_{Z}(t)=A_{2} \sin \left(\omega t-\phi_{0}\right)$

in which $U_{\theta}(t)$ is the tangential displacement and $U_{Z}(t)$ is the longitudinal displacement on the stator surface. $A_{1}$ and $A_{2}$ represent the amplitudes of tangential and longitudinal displacements, respectively. $\phi_{0}$ is the temporal phase shift between the tangential and longitudinal displacements and $\omega$ is the angular frequency.

Through Eqs. (1) and (2), the trajectory equation of a point on the stator surface reads

$\frac{U_{\theta}^{2}(t)}{A_{1}^{2} \sin ^{2} \phi_{0}}-\frac{2 \cos \phi_{0}}{A_{1} A_{2} \sin ^{2} \phi_{0}} \cdot U_{\theta}(t) \cdot U_{Z}(t)+\frac{U_{Z}^{2}(t)}{A_{2}^{2} \sin ^{2} \phi_{0}}=1$

Eq. (3) shows that the trajectory of a point on the stator surface depends on the temporal phase shift $\phi_{0}$, amplitudes $A_{1}$ and $A_{2}$. If amplitude $A_{1}$ equals to $A_{2}$, the trajectory will differ from the temporal phase shift $\phi_{0}$. In the case that $\phi_{0}$ is $\pi / 2$, the trajectory forms a circle. On the other hand, if the temporal phase shift $\phi_{0}$ keeps the value $\pi / 2$, the trajectory will depend on various ratios $A_{1} / A_{2}$ (see Fig. 2).

The working principle of the present motor can be illustrated by Fig. 3. The temporal phase shift $\phi_{0}$ is as-

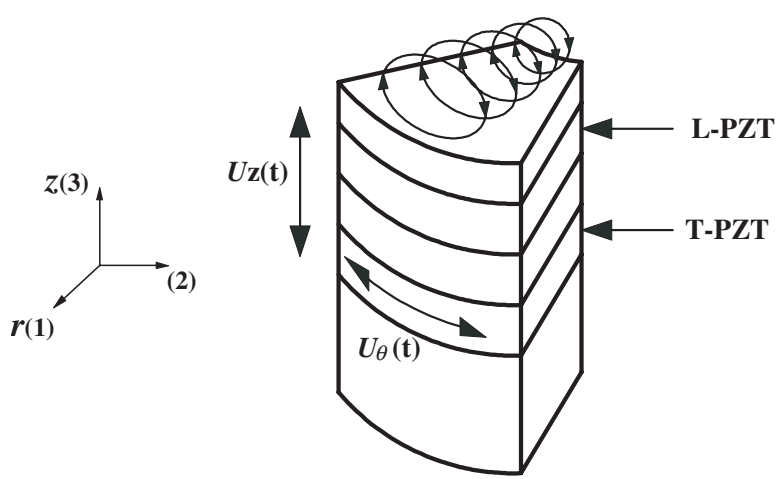

Fig. 2. Elliptical locus on the stator surface. 

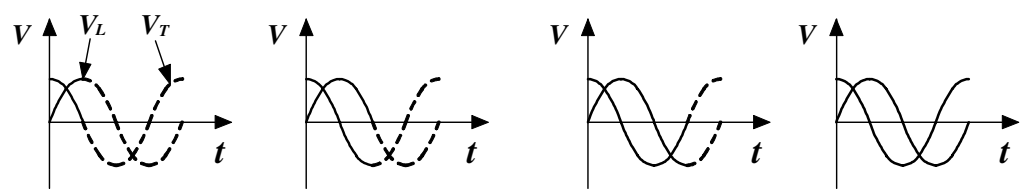

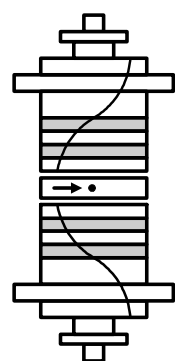

(a)

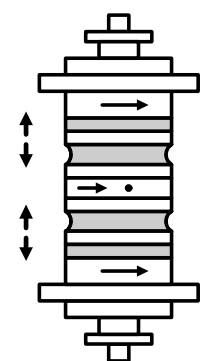

(b)

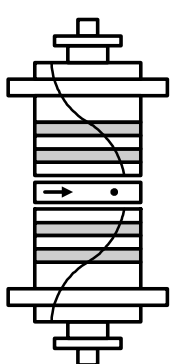

(c)

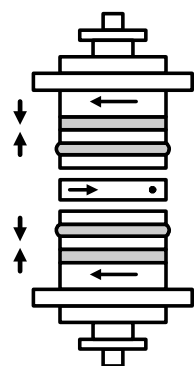

(d)

Fig. 3. Working principle of motor.

sumed $\pi / 2$. When the voltage $V_{\mathrm{T}}$ applied to T-PZT reaches its peak value, the torsional displacement obtains the maximum while the longitudinal transducer has no deformation. Two stators are in loose contact with the rotor (see Fig. 3a). As the voltage $V_{\mathrm{L}}$ applied to L-PZT increases and reaches the peak, the longitudinal transducer extends and two stators are in firm contact with the rotor (see Fig. 3b). At this moment, the tangential velocity of points at the interface between rotor and stator reaches the maximum, and the rotor will rotate undergoing the friction forces. In Fig. 3c, the torsional deformation of the stators will be along the same direction, but the longitudinal transducer will contract and its deformation decreases to zero. Two stators are in loose contact with the rotor again. Finally, the longitudinal transducer will continue to contact while the torsional transducer begins to deform in reverse direction, and the rotor will separate from two stators. Due to the inertia, the rotor will rotate in the same direction (see Fig. 3d). In such a cycle, the rotor has rotated by a small angle. With the high frequency $\mathrm{AC}$ voltages excitation, a continuous rotary motion occurs in this motor.

\section{Resonant frequencies coincidence}

The previous longitudinal and torsional ultrasonic motor usually works at the resonance frequency of the torsional vibration. In order to obtain a larger output torque and a higher efficiency, however, both the torsional transducer and the longitudinal transducer should be driven in resonance frequency. Theoretically, the eigenfrequency of longitudinal vibration is not equal to that of torsional vibration within a cylinder motor because of difference propagation speed of these two waves.
The present motor uses two stators and two adjusting rings to match the resonance frequencies of both transducers. To analyze properties of the motor and the functions of the rings, a FE model of stator is developed and a commercial software ANSYS is used. Because of the symmetrical structure, only one stator is used for FE modeling (see Figs. 4 and 5). The physical models of these two vibrations have the same geometry, but the

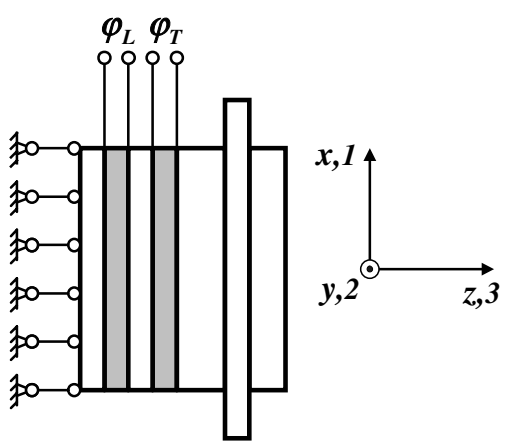

Fig. 4. Physical model for longitudinal vibration.

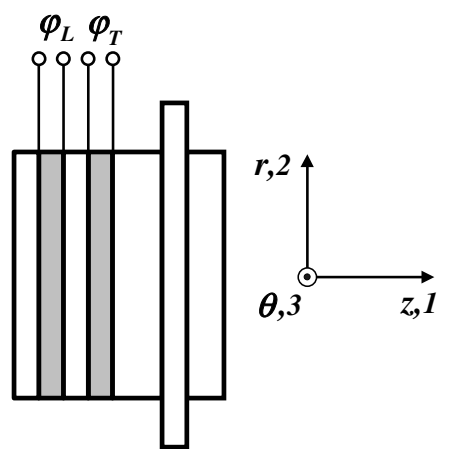

Fig. 5. Physical model for torsional vibration. 


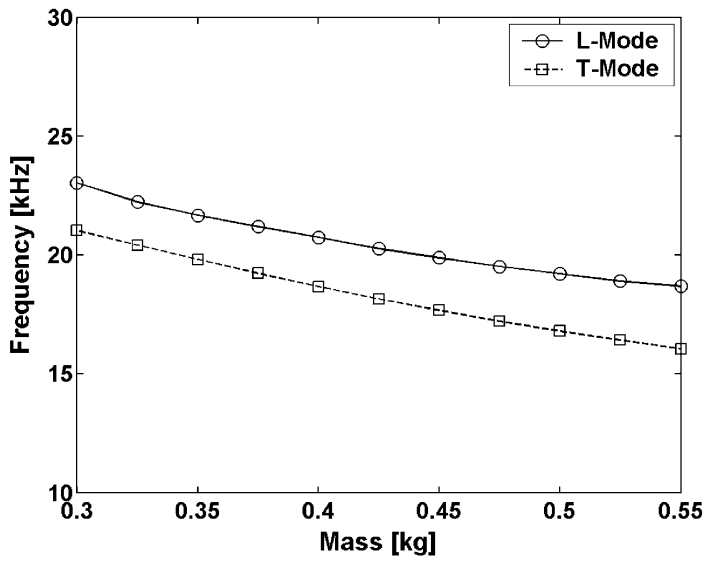

Fig. 6. Eigenfrequencies for changing mass of the ring using FE model.

coordinate systems and the boundary conditions are different. The direction of electric field for the longitudinal transducer is the same as that of the polarization, while the direction of electric field for the torsional transducer is orthogonal to that of the polarization.

It is assumed that two electrodes of the PZT are connected (short-circuited). For the physical model of longitudinal vibration, it is considered that one end of stator is fixed and the other is free. For the physical model of torsional vibration, both ends of the stator are free. To match the eigenfrequencies of both vibrations, two cases are considered: one is to change the mass of the ring and the other is to change the position of the ring.

Fig. 6 shows that the first-order eigenfrequency of longitudinal vibration decreases slightly with an increase of the mass of the ring. This is due to the well-known fact that the eigenfrequency is in counter proportion with the mass. The eigenfrequency of longitudinal and the eigenfrequency of torsional vibration have the same tendency when the mass of the ring is changed. There-

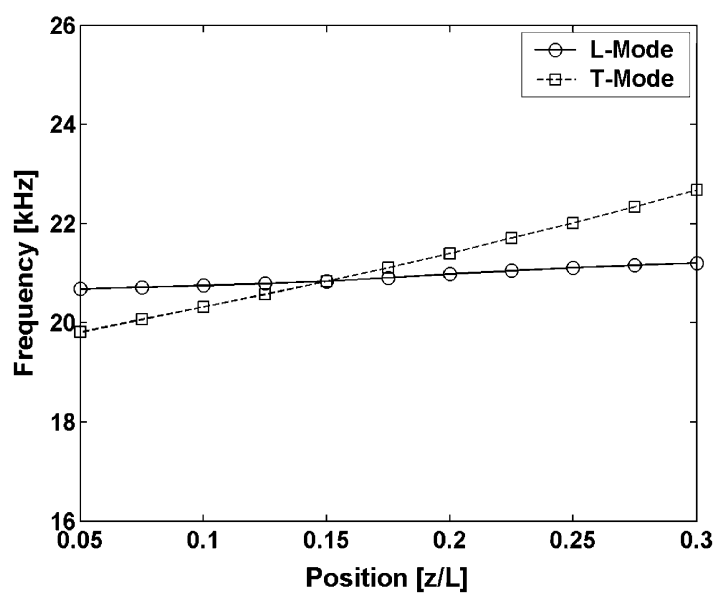

Fig. 7. Eigenfrequencies for changing position of the ring using FE model. fore, it is impossible to obtain better effect of matching the two eigenfrequencies only via changing the mass of the ring.

Fig. 7 shows that the first-order eigenfrequency of the longitudinal vibration is almost independent of the position of the ring while the first-order eigenfrequency of the torsional vibration is much affected by this factor. When the rings are moved to a certain position, the eigenfrequency of longitudinal vibration has the same value as that of trosional vibration and the resonance frequency of both vibrations is $20.75 \mathrm{KHz}$. Obviously, this property can be used to match the eigenfrequencies of longitudinal and torsional vibrations.

\section{Experimental investigations}

The geometries of the present motor are optimized based on the FE analytical results and a prototype of this motor is fabricated (see Fig. 8). The length of the motor is $120 \mathrm{~mm}$, the outside diameter of the stator is $40 \mathrm{~mm}$ and the outside diameter of the adjusting ring is $60 \mathrm{~mm}$. Two ring-shaped piezoelectric ceramics with an inside diameter of $25 \mathrm{~mm}$, an outside diameter of $40 \mathrm{~mm}$ and a thickness of $4 \mathrm{~mm}$ are used as the longitudinal transducers in two stators for generating the longitudinal vibration. Two layers of rectangle-shaped shear PZT in a stator are bonded with an epoxy adhesive forming a ring shape and generating the torsional vibration. A ring-shaped ceramic with the same size as L-PZT is used as an isolator, which is placed between two transducers.

Fig. 9 shows the scheme of experimental setup. A signal generator is connected via a phase-shifter to two amplifiers for excitation of longitudinal vibration and torsional vibration. The phase-shifter allows phase shift between 0 and $\pi$. The amplitudes of two exciting voltages and the phase shift between them can be adjusted separately to control the rotary speed and the direction

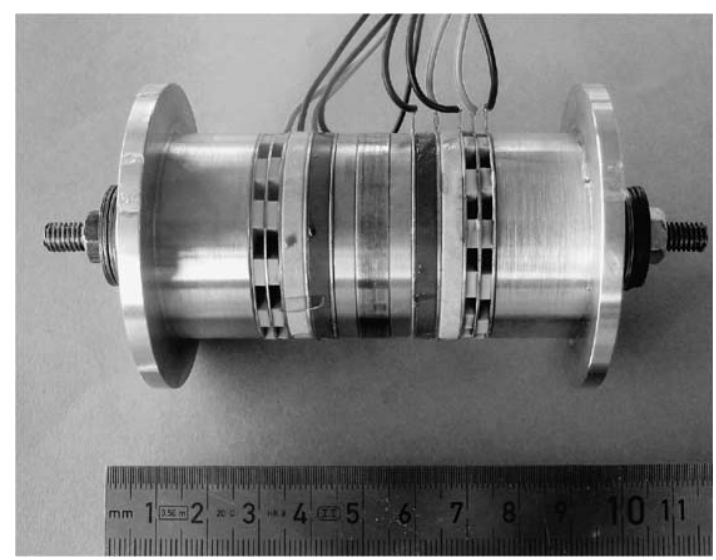

Fig. 8. The prototype of present motor. 


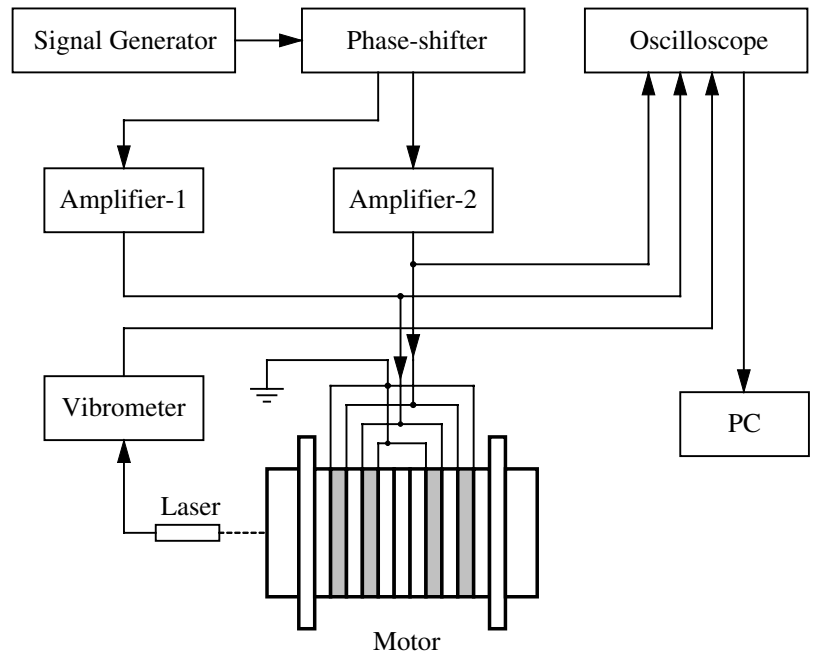

Fig. 9. The scheme of experimental setup.

of the rotor. In experiments, the eigenfrequencies of longitudinal vibration and torsional vibration are excited by two voltages with 90 degrees temporal phase shift between the two vibrations. The eigenfrequencies and eigenforms of these two vibration modes are measured by laser vibrometer that are $19.48 \mathrm{KHz}$ and $17.64 \mathrm{KHz}$
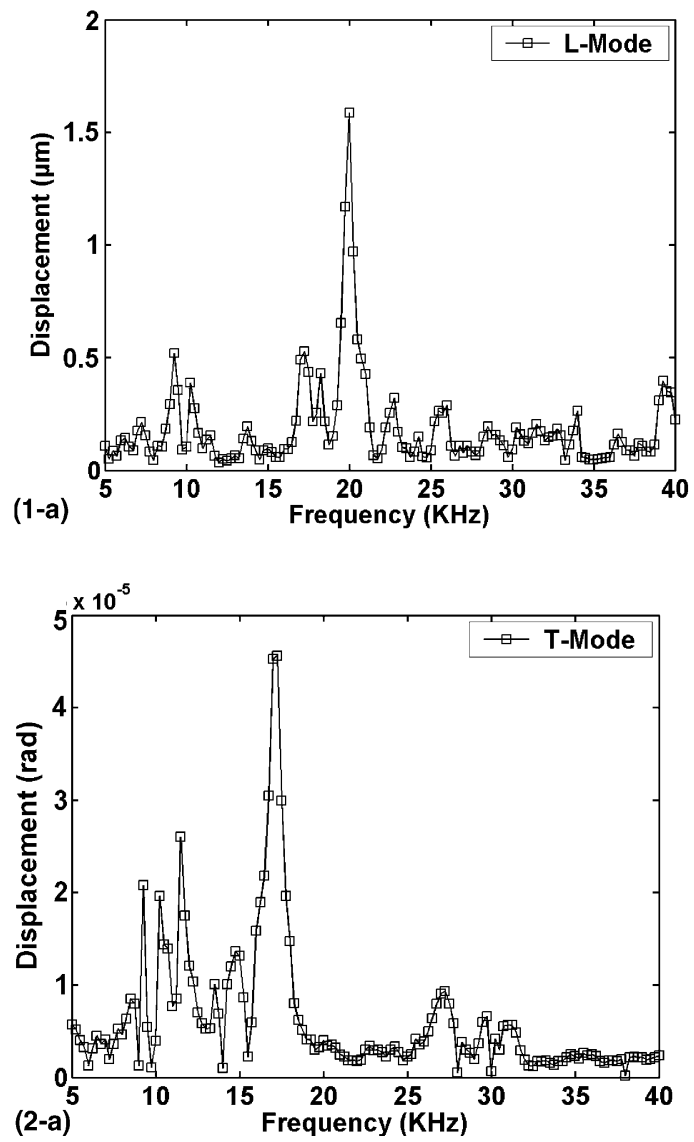

respectively when the ring is placed at the end of the stators (see Fig. 10).

The load characteristic of the present motor was measured under three different exciting voltages for two transducers (see Fig. 11). The rotary speed decreases greatly with the load increasing. Under no load condition, the motor behaves as the theoretical predictions

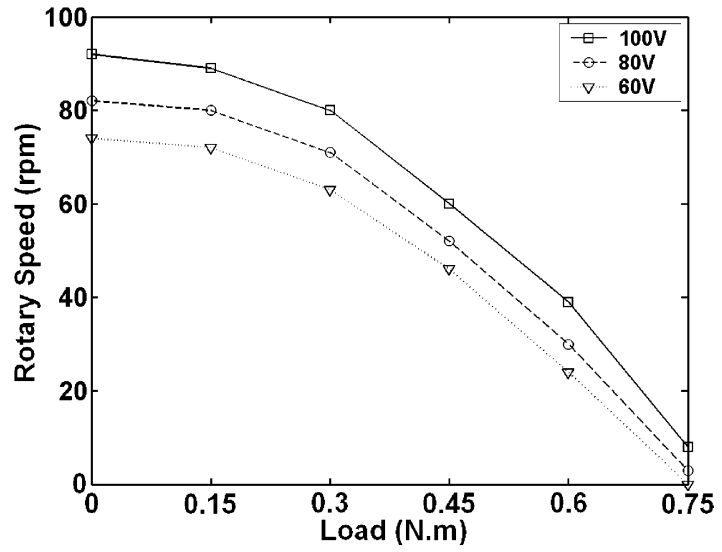

Fig. 11. Rotary speed versus load for the motor under different exciting voltages.
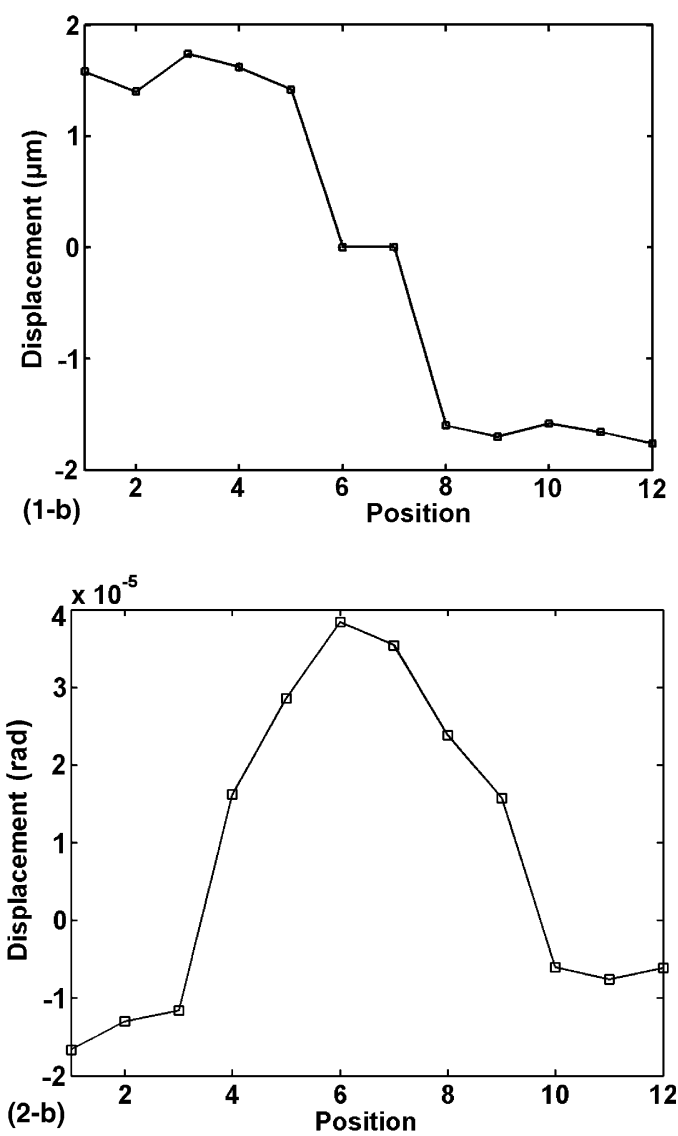

Fig. 10. The measured first-order eigenfrequency of two vibration modes. (1) Longitudinal vibration mode: (1-a) Eigenfrequency and (1-b) eigenform. (2) Torsional mode: (2-a) Eigenfrequency and (2-b) eigenform. 
and the speed of rotor reaches the maximum $92 \mathrm{rpm}$ at the working frequency $19.0 \mathrm{KHz}$. Compared with the FE analytical value $20.75 \mathrm{KHz}$, the experiment value $19.0 \mathrm{KHz}$ has about $8.4 \%$ error. Considering the glue used to stick the PZT is neglected in FE model, this error could be acceptable. To obtain excellent characteristics, the present motor should be improved in the future.

\section{Conclusions}

A new longitudinal and torsional piezoelectric ultrasonic motor with two stators is developed in this paper. The symmetrical structure of the present motor as well as two adjusting rings on the stator can be utilized to match the resonance frequencies of longitudinal and torsional vibrations to obtain large output torque. A FE model of stator is proposed and the analytical results show that through changing the position of the rings the first-order resonance frequency of longitudinal vibration is coincided with that of torsional vibration. In the experiment the present motor behaves as the theoretical predictions and the measured first-order eigenfrequencies of longitudinal and torsional vibrations are $19.48 \mathrm{KHz}$ and $17.64 \mathrm{KHz}$, respectively. The speed of rotor reaches the maximum $92 \mathrm{rpm}$ at the working frequency $19.0 \mathrm{KHz}$.

\section{Acknowledgement}

This paper has benefited from the experiment with Dipl.-Ing. Andreas Maier, whose help is gratefully acknowledged.

\section{References}

[1] T. Sashida, T. Kenjo, An Introduction to Ultrasonic Motors, Clarendon Press, Oxford, 1993.

[2] S. Ueha, Y. Tomikawa, Ultrasonic Motors-theory and Applications, Clarendon Press, Oxford, 1993.
[3] S. Ueha, Present status of ultrasonic motors, IEEE Ultrasonic Symposium (1989) 749-753.

[4] K. Nakamura, M. Kurosawa, S. Ueha, Characteristics of a hybrid transducer-type ultrasonic motor, IEEE Transactions on Ultrasonics, Ferroelectrics, and Frequency Control 38 (3) (1991) 188-193.

[5] M. Kurosawa, S. Ueha, Hybrid transducer type ultrasonic motor, IEEE Transactions on Ultrasonics, Ferroelectrics, and Frequency Control 38 (3) (1991) 89-92.

[6] K. Nakamura, S. Ueha, Performances of a hybrid transducer type ultrasonic motor as a function of the size, 1994 Ultrasonics Symposium, New York, 1994, pp. 557-560.

[7] Lin Shuyu, Study on the sandwiched piezoelectric ultrasonic torsional transducer, Ultrasonics 32 (6) (1994) 461-464.

[8] K. Nakamura, M. Kurosawa, S. Ueha, Design of a hybrid transducer type ultrasonic motor, IEEE Transactions on Ultrasonics, Ferroelectrics, and Frequency Control 40 (4) (1993) 395 401.

[9] T. Isjii, T. Shinkoda, S. Ueha, K. Nakamura, M. Kurosawa, Efficiency improvement of an ultrasonic motor driven with rectangular waveform, Japan Journal of Applied Physics 35 (5B) (1996) 3281-3285.

[10] J. Satonobu, J.R. Friend, K. Nakamura, S. Ueha, Numerical analysis of the symmetric hybrid transducer ultrasonic motor, IEEE Transactions on Ultrasonics, Ferroelectric, and Frequency Control 48 (6) (2001) 1625-1631.

[11] Tomikawa, K. Adachi, M. Aoyagi, T. Sagae, T. Takano, Some constructions and characteristics of rod type piezoelectric ultrasonic motors using longitudinal and torsional vibrations, IEEE Transactions on Ultrasonics, Ferroelectric, and Frequency Control 39 (5) (1992) 600-608.

[12] W. Seemann, R. Gausmann, Transmission and reflection coefficients for longitudinal waves obtained by a combination of refined rod theory and FEM, Journal of Sound and Vibration 197 (5) (1996) 571-587.

[13] J. Satonobu, D.K. Lee, K. Nakamura, S. Ueha, Improvement of the longitudinal vibration system for the hybrid transducer ultrasonic motor, IEEE Transactions on Ultrasonics, Ferroelectric, and Frequency Control 47 (1) (2000) 216-221.

[14] M. Aoyagi, Y. Tomikawa, Improvement in performance of longitudinal and torsional vibrator combination-type ultrasonic motor, Japan Journal of Applied Physics 38 (5B) (1999) 3342 3346.

[15] M. Aoyagi, Y. Tomikawa, New designed longitudinal and torsional vibrator combination-type ultrasonic motor, Ferroelectrics 232 (1999) 241-246.

[16] Zhou Tieying, Zhang Liqun, Xue Yi, Chenye, Resonant frequencies into degeneration of hybrid longitudinal and torsional ultrasonic motor vibration system, Piezoelectrics \& Acoustooptics 21 (2) (1999) 150-160. 